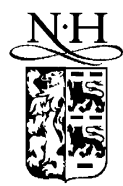

ELSEVIER

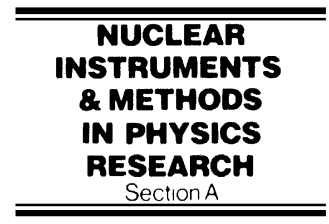

www.elsevier.com/locate/nima

\title{
Radioactive source control and electronics for the ATLAS tile calorimeter cesium calibration system
}

\author{
N. Shalanda, A. Karyukhin*, S. Kopikov, A. Shalimov, \\ M. Soldatov, A. Solodkov, E. Starchenko \\ Institute for High Energy Physics, Protvino 142281, Russia \\ For the ATLAS Tile Calorimeter Collaboration \\ Received 15 April 2003; accepted 8 May 2003
}

\begin{abstract}
A system using a radioactive ${ }^{137} \mathrm{Cs}$ source to calibrate and monitor the Hadron Calorimeter (TileCal) of the ATLAS experiment at the LHC is described. The system includes a set of sensors to monitor the position of the source which moves via hydraulic propulsion. The design of the sensors, the corresponding electronic modules and their performance are detailed.
\end{abstract}

(C) 2003 Elsevier B.V. All rights reserved.

PACS: $07.07 . \mathrm{Df} ; 29.25 . \mathrm{Rm} ; 29.40 . \mathrm{Vj}$

Keywords: Calorimeter; Calibration; Monitoring; Movable radioactive source; Sensor

\section{Introduction}

ATLAS [1] is a general-purpose pp-experiment at the Large Hadron Collider (LHC) under construction at CERN. The Hadron Calorimeter (TileCal) together with the Liquid Argon electromagnetic calorimeter (LAr) comprise the ATLAS calorimeter system as shown in Fig. 1.

The TileCal is a sampling calorimeter constructed of steel plates (the absorber) and scintillating plastic tiles (the active material). The design, general features and expected performance of the

\footnotetext{
*Corresponding author.

E-mail address: karyukhin@mx.ihep.su (A. Karyukhin).
}

calorimeter are given in the "ATLAS Tile Calorimeter Technical Design Report" [2].

The TileCal is divided into one barrel (TLB) and two extended barrel (TLE) sections. All three sections have a cylindrical structure with an inner radius of $2280 \mathrm{~mm}$ and an outer one of $4230 \mathrm{~mm}$. The TLB section is $5640 \mathrm{~mm}$ in length along the beam axis, while each TLE section has a length of about $2910 \mathrm{~mm}$.

Each of the cylinders is further subdivided into 64 independent azimuthally oriented modules. Within each module, there are a number of geometric cells read out via optical fibres.

To calibrate and monitor the TileCal, a movable $8-9 \mathrm{mCi}{ }^{137} \mathrm{Cs} \gamma$-source is used with energy $E_{\gamma}=$ $0.662 \mathrm{MeV}$. The use of a movable source allows to 


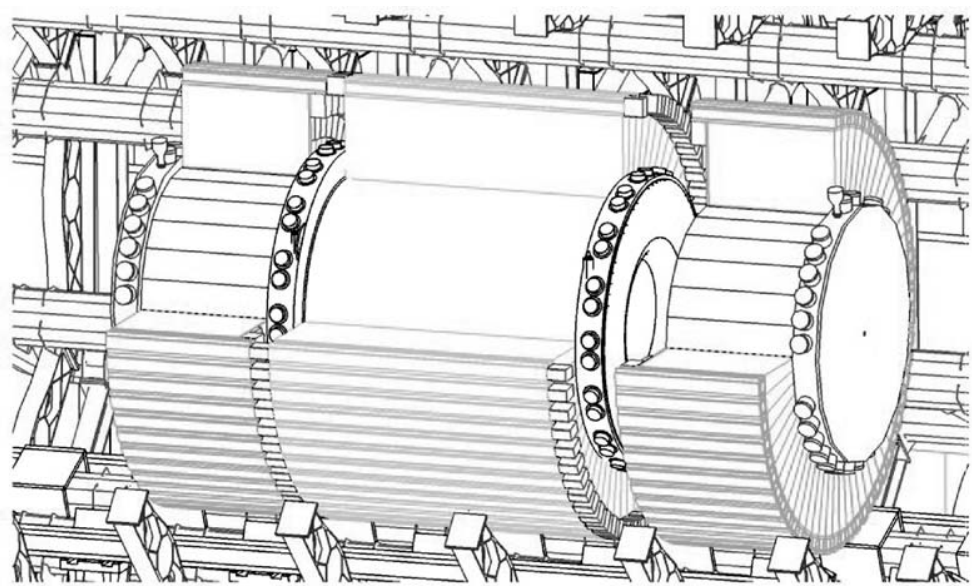

Fig. 1. Schematic view of the ATLAS calorimeter system. The three TileCal cylinders (segmented in azimuth) surround the LAr calorimeter modules.

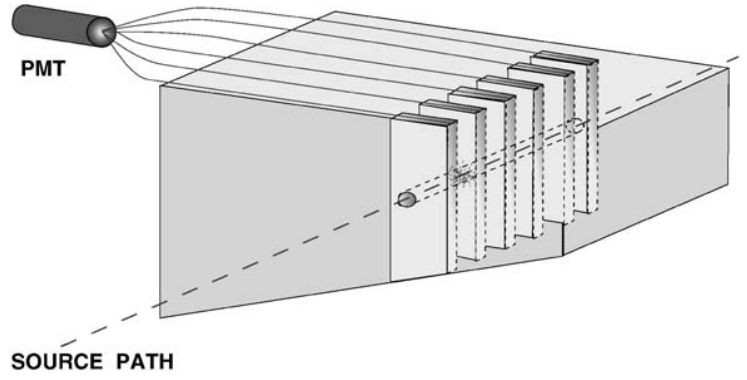

Fig. 2. Schematic view of the ${ }^{137} \mathrm{Cs}$ source calibration system. The source passes through all the tiles of the calorimeter.

test the optical quality of the scintillators and fibres, to equalize the response of all the cells, to monitor each cell over the time and to provide the overall energy calibration.

The basic concept showing the source path through a number of tiles is given in Fig. 2. When each tile is traversed by the ${ }^{137} \mathrm{Cs}$ source, $\gamma$-quanta induce scintillating light emission in the tile, which is transmitted via an optical fibre to a photomultiplier tube (PMT). The magnitude of the PMT current depends upon the tile material and the optical quality of the fibre [3].

The correlation of the induced current (or "PMT response") as a function of the source position is shown in Fig. 3. The multiple peak structure in the diagram is due to the passage of the source through individual tiles; a case of faulty

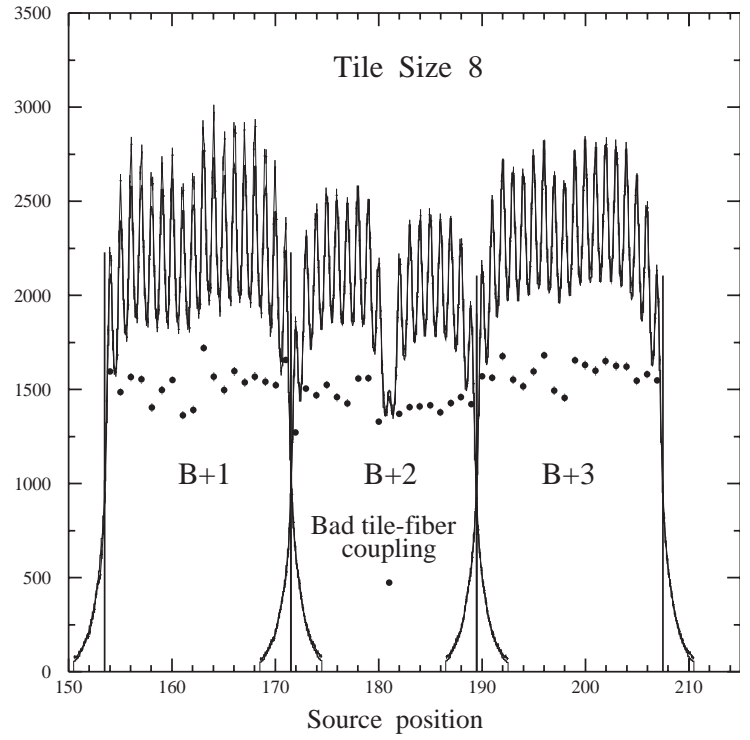

Fig. 3. PMT current as a function of the source position in a cell.

light collection from one fibre is clearly seen. This figure demonstrates the power of source scans of the calorimeter in testing of its quality and uniformity.

The capability to resolve individual tiles is due to the mean-free path of the gammas, which is of the order of the $18 \mathrm{~mm}$ separation between tiles. The source can "see" every scintillating tile, and, 
therefore, it can be used to obtain an "X-ray picture" of the response.

\section{Source drive concept}

In order to transport the ${ }^{137} \mathrm{Cs}$ source through all the 192 (64 TLB and 128 TLE) modules of the TileCal, an elaborate source drive and monitoring system control are needed. Such a system (MonSys) has been designed $[2,6]$ where the ${ }^{137} \mathrm{Cs}$ source is driven by a flowing liquid (water) through a series of calibration (or "source") tubes which pass through all the cells of the calorimeter.

A stainless-steel tube of $6.0 \mathrm{~mm}$ inner diameter and $8.0 \mathrm{~mm}$ outer diameter is used to guide a capsule containing the source. Three independent calibration pipeline circuits are necessary, one for the TLB section and two for the TLE ones. In the radial direction of the TileCal there are 11 tile layers, so that 11 tubes connected by joint sections are necessary for each module. The total tube length required to cover all the TileCal modules is about $10 \mathrm{~km}$.

The source capsule, as shown in Fig. 4, is made of hardened aluminium alloy coated with titanium nitride (TiN), which surrounds the source, contained in a small welded stainless-steel capillary [4]. The dumb-bell shape and the outside dimensions of the capsule permit to drive it through curved tube sections with a bending radius down to $15 \mathrm{~mm}$.

A set of specially designed sensors is needed in order to control and monitor the capsule movement inside the long pipeline system. The sensors

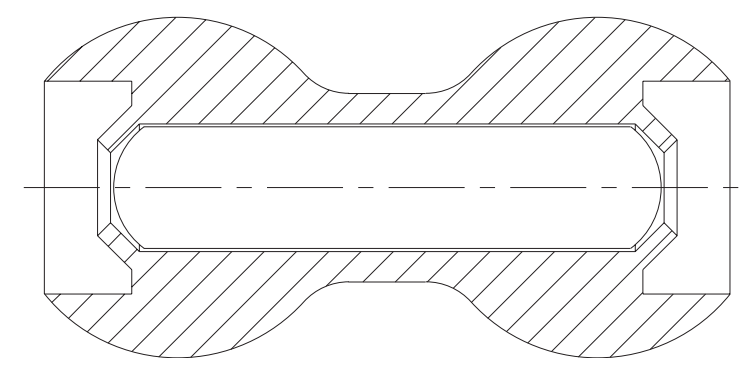

Fig. 4. Schematic view of the source capsule. must provide information on the capsule position at a given time and place (at the storage position, for example) or simply register that it has passed a particular point in order to follow its progress.

Taking into account the operating conditions of the monitoring system, the reliability and safety aspects, the sensors mounted on tubes should satisfy the following requirements:

- They must be able to measure the capsule velocity over a wide range, up to $50 \mathrm{~cm} / \mathrm{s}$ (the nominal capsule speed is about $30 \mathrm{~cm} / \mathrm{s}$ ).

- There should be no interference with the movement of the capsule, to ensure the reliability of the pipeline (e.g., not to increase the risk of leaks).

- The sensors should be capable of detecting both a radioactive capsule and an empty one (dummy source), to allow system tests and verification.

- The sensors must be reliable and require minimal maintenance.

- No electronic noise should be generated.

- The sensors must be radiation tolerant and be able to operate in a strong magnetic field.

Assuming that at least two sensors are needed for a module (one for each entry and one for exit point) the total number of sensors for the whole system is about of 400 pieces. Two types of sensors have been developed: sensors based on optical detection (IRS) and sensors based on electromagnetic detection (source location sensor (SIN)). Some test results for both types of sensors have been described in Ref. [5].

\section{IRS-“infra red sensors"}

The optical detection principle by means of the IRS sensor is built on an emitter-sensor pair integrated into the mechanical connector at the straight to bent joint section as shown in Fig. 5. The light emitter is an infrared LED (AL107) operating at a wavelength of $950 \mathrm{~nm}$. The receiver is a phototransistor FT-2B (Russian specifications). Two plastic lenses imbedded in the sensor body help to focus the light according to the source capsule shape. 


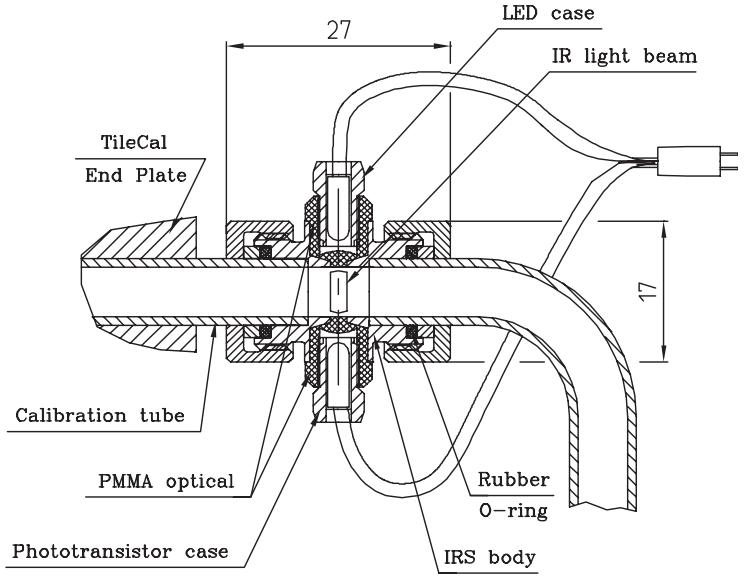

Fig. 5. Schematic view of the IRS sensor.

The interruption of a pulse train indicates the passage of the capsule and allows its velocity to be measured. The IRS sensor readout electronics is designed to register the precise moment of a capsule passage ("event"). One IRS electronics chain supports up to 16 channels and consists of two modules: the analogue module (IRS_A), situated no more than $5 \mathrm{~m}$ away from the sensors, and the digital module (IRS_D), located at some distance in a CAMAC crate in the control room. Both modules communicate via a serial connection on a cable which can be over $50 \mathrm{~m}$ in length. The cable provides low voltage power, so the analogue module does not require an additional power supply.

The analogue module IRS_A consists of a channel number (address) decoder, an LED pulse driver, photocurrent amplifiers and comparators, an output signal multiplexer. Input address lines select the active sensor number. The photocurrent signals pass through an amplifier and shaper, and are combined into one output signal. The output current for the 16 sensors in a chain can be adjusted with an on-board trimmer, to be in the range from 10 to $100 \mathrm{~mA}$.

The digital module IRS_D conforms to the CAMAC standard, and includes an internal quartz frequency generator, a mask register/multiplexer, a noise suppression filter, a 28-bit time counter, an event register, and an event trigger.
The module generates an event record containing the sensor number and the time, with a typical time resolution of $1 \mathrm{~ms}$.

Several IRS_D modules can be synchronized with common "Clock" and "Clear" signals in order to provide an absolute event time reference. Using a jumper switch, one can select the internal clock frequency of the module, giving a time resolution of $0.5,1,2$ or $4 \mathrm{~ms}$. An external frequency generator can be used for the clock signal distribution from one "master" module. Channels which are unused or broken can be disabled by a mask register.

False signals generated by contamination in the liquid, such as air bubbles suspended in the water, can be a serious problem for any optical sensor including the IRS. In order to suppress such spurious signals, the IRS_D module is equipped with a special digital noise suppression filter. The principle of the filtering is illustrated in Fig. 6.

For example, every $1 \mathrm{~ms}$ an LED emits a light pulse of duration $\frac{1}{16} \mathrm{~ms}$. The same succession of pulses is produced by all IRS modules. The resulting photocurrent measured in the optocoupler channel corresponds to a " 0 " state, while the absence of a pulse corresponds to a " 1 " state. The series of pulses reflects the profile of any opaque object, such as the capsule, as it passes by a sensor.

The last five measurements of the sensor are stored in a shift register and then compared with a predetermined pattern. Example patterns are (1), (11), (111), (1111), (01), (011), (0111) or (01111).
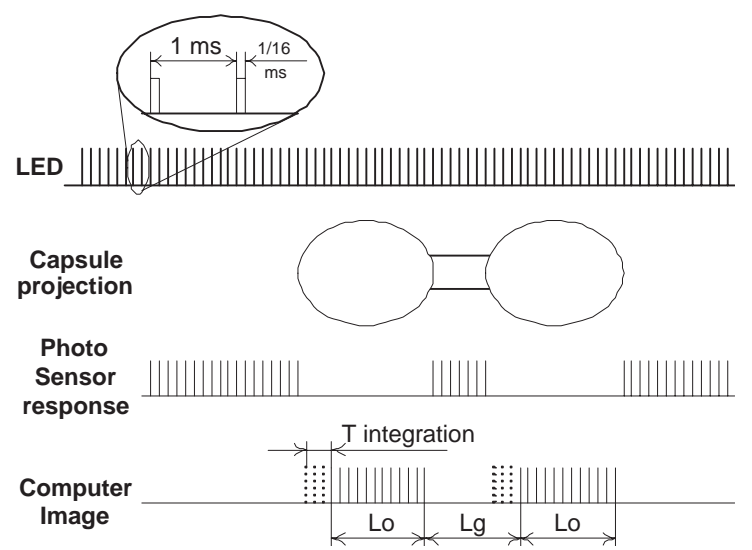

Fig. 6. Noise suppression algorithm used in the IRS_D module. 
Fig. 6 illustrates the combination (1111), labelled here as a "T integration", which requires at least four sequential LED pulses to be blocked in order to be classified as an "event". As a result of this requirement, objects which block the LED pulses for less than $4 \mathrm{~ms}$ are ignored; this is sufficient to suppress fake signals produced by small air bubbles. This requirement can be tightened further by demanding a pattern such as (01111), which registers the initial light pulse just before the capsule edge.

The middle narrow section of the capsule does not completely block the LED light and as a result the capsule appears to the sensors as two separate objects having equal length, $L_{\mathrm{o}}$, which pass the sensor one after the other. This behaviour provides an additional handle on noise suppression. By measuring the time delay, $L_{\mathrm{g}}$, between the signals from the two large capsule sections, one can either measure the capsule length or use this information for further event triggering.

A 32-bit "Event Register" is used to store a 4-bit channel number and a 28-bit event time. If an event occurs, an Event Trigger flag will be set, and no further events will be recorded until the Event Register is read out or a CLEAR signal is received. The Event Trigger status can be tested by checking either the CAMAC "LAM" signal or the CAMAC "Q" signal during the reading of the Event Register. The current status of the Event Trigger is indicated on a front panel LED.

During the period 1995-1997 several MonSys prototypes with IRS sensors were studied. The stability of the capsule motion, the operation of the pump and valves, the performance of other equipment and control procedure sequences were thoroughly tested. The results of these studies provided a knowledge base and laid the foundation for the final system construction.

In the years 1996-1997, during test beam periods for the TileCal full-scale prototype "Module 0" at CERN, the hydraulic calibration system was equipped with more than 50 IRS sensors and the corresponding number of electronic modules. All equipment operated reliably with negligible electronic noise.

However, the IRS system has one very important disadvantage, namely the need of drilling and fixing many open optical channels in the tubes. It turns out to be impractical to install so many sensors in a big pipeline system, in order to avoid risks to the tube hermeticity. As a result, for the elaborate tube system necessary for ATLAS, the IRS sensor approach was discarded in favour of a second system, described below.

\section{SIN-"sensors based on inductance"}

A sensor based on an inductance measurement was proposed to record the source capsule movement. The sensor itself is a coil located on a tube in such a way that a part of the alternating magnetic flux generated by the coil permeates the tube's inner radius, as shown in Figs. 7 and 8.

The coil is a part of an LC circuit which operates continuously. The presence of the capsule causes a change in the coil inductance, resulting in a variation of the oscillation frequency, which can be detected and used as a trigger signal.

Several coil types with different inductance values have been tested. A conventional stainlesssteel tube with an external diameter of $8 \mathrm{~mm}$ and a wall thickness of $1 \mathrm{~mm}$ has been used.

A typical dependence of the frequency variation of the oscillator's frequency when a capsule passes the coil is illustrated in Fig. 9, which compares the signals from an aluminium and a titanium capsules. For the aluminium capsule, the maximum effect was achieved with the LC-generator

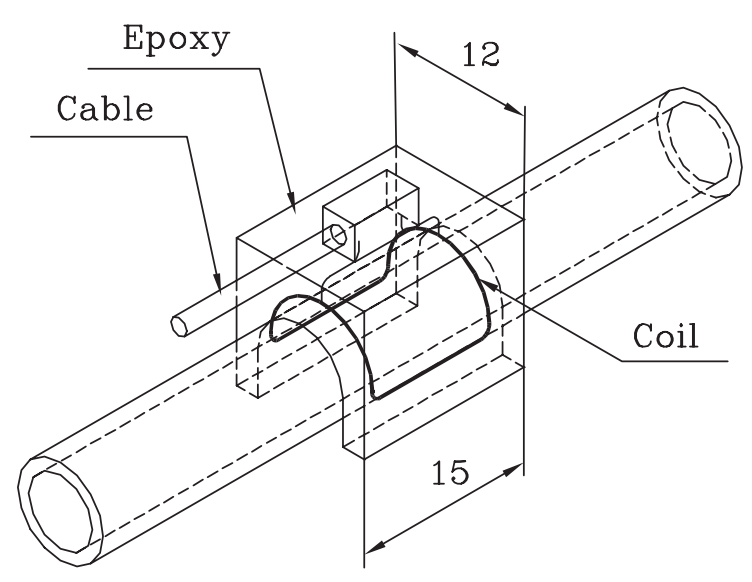

Fig. 7. Design view of a SIN sensor. 


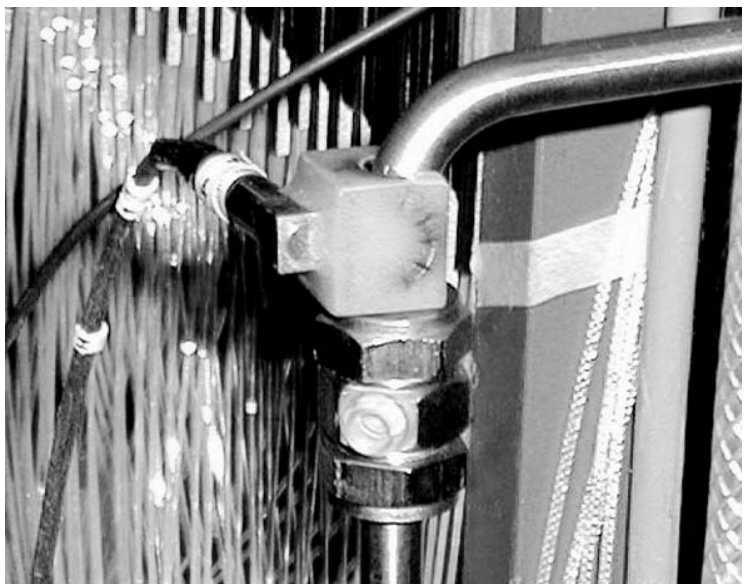

Fig. 8. A SIN sensor in a test bench setup installed on a bent section of the source tube.

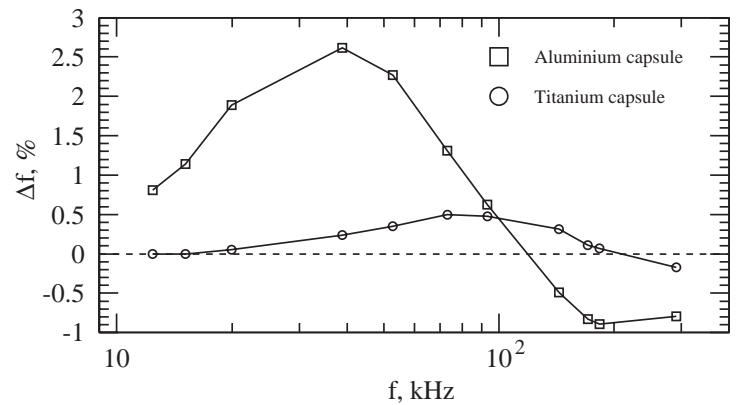

Fig. 9. Coil frequency variation generated by the presence of a source capsule. Aluminium and titanium capsules of the same size and shape were used.

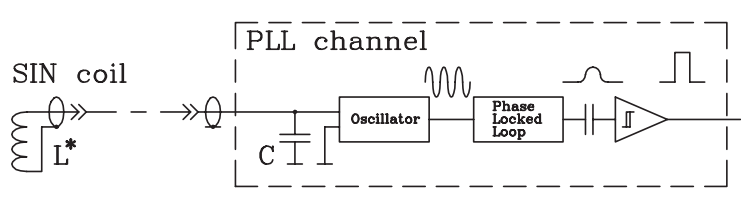

Fig. 10. A single channel of the SIN_C Module.

frequency operating between 30 and $40 \mathrm{kHz}$. The titanium capsule was found to have a much smaller influence upon the oscillator's frequency.

One SIN electronic module of an early version of "SIN_C" is shown in Fig. 10. It supports up to 16 sensor channels, and consists of 16 independent Phase Locked Loop (PLL) channels and shapers along with a CAN-bus interface.
To measure frequency variations an integrated PLL circuit KR1561GG1 (Russian analogue of CD4046B by Micropower) was used. External components (two resistors and one capacitor) of the PLL circuit provide a capture and tracking range of $10-50 \mathrm{kHz}$, corresponding to a sensitivity of about $200 \mathrm{mV} / \mathrm{kHz}$. The LC-generator frequency was chosen close to the upper limit of the range of $40 \mathrm{kHz}$, at the peak sensitivity. The resolution of the capsule tracking was of the order of $10-15 \mathrm{~ms} / \mathrm{kHz}$.

Another method for tracking the capsule is the direct frequency measurement of the generator oscillations. This is implemented in the "SIN_CAN" latest version of the sensor electronic module.

The advantages of this solution are the low power consumption, a compact package, no need for external adjustable components and the low cost. A SIN-CAN module schematic is shown in Fig. 11.

Shaped pulses at fixed time interval are counted and the number is compared to a reference. The non-trivial real-time algorithm of pulse counting and threshold tracking for 16 input channels is implemented in a RISC microcontroller of the Atmel AVR family. The microcontroller masks unused channels, evaluates reference levels, and filters slow and rapid frequency changes. The system can also measure the base frequency of each channel, as well as read and modify the microcontroller program via the CAN-bus.

An example of a single-module calibration setup in a test bench is given in Fig. 12. In this case, 16 SINs give the position of the source as it passes through the module. The other sensors in the

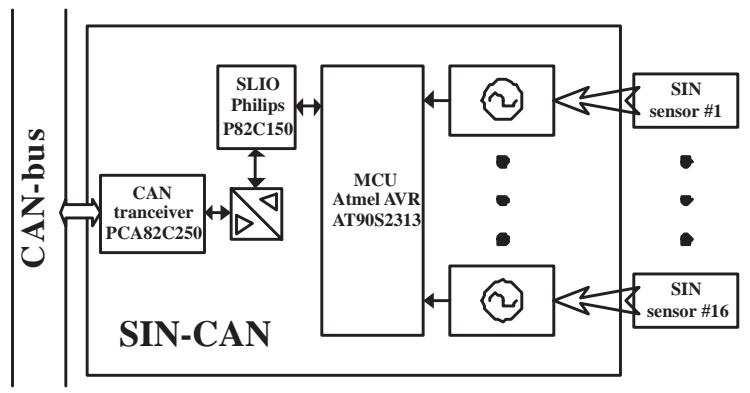

Fig. 11. The SIN-CAN module concept. 


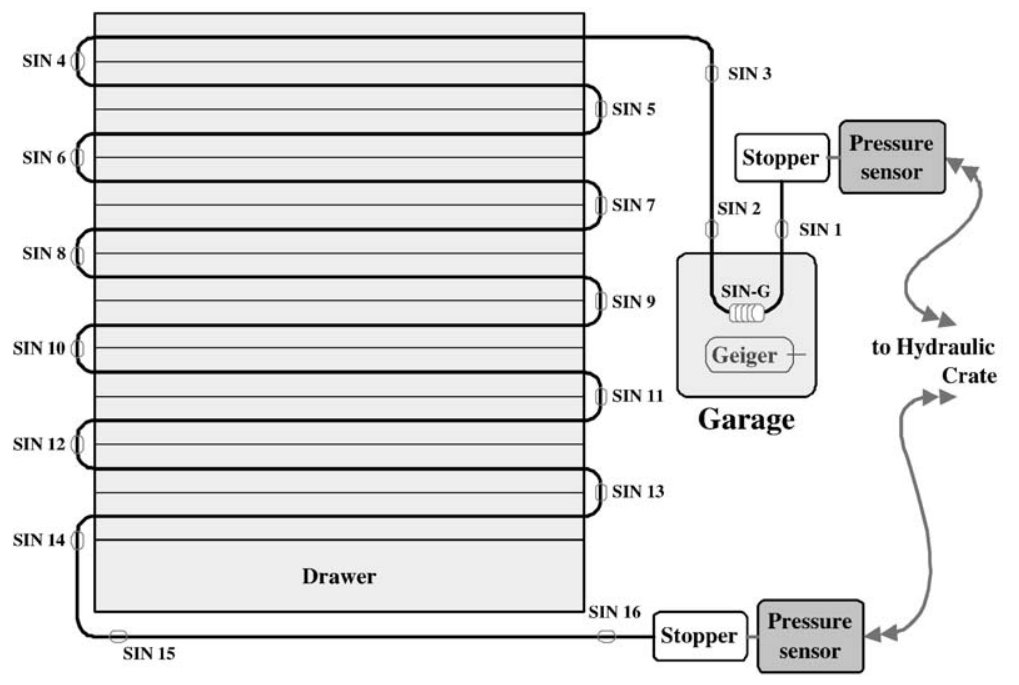

Fig. 12. An example layout of the calibration tubes and sensors.

system are a Geiger counter and pressure sensors described below.

During field tests in 1997-2000, the SIN sensor system demonstrated low sensitivity to noise, robustness and excellent reliability. Starting from 1999 onwards, SIN sensors have been used in all active MonSys prototypes at CERN (three systems in total).

Note that both types of sensors, IRS and SIN, were tested in a magnetic field up to $1 \mathrm{~T}$. The results of the tests showed the sensors' performance to be independent of the magnetic field.

\section{Garage- "source storage device" sensors}

The "Garage" is the name given to a mechanical device in which the source is stored between calibration runs. The garage must be capable of accepting a source capsule, recognize its presence and lock or release the capsule whenever it is needed according to a remote control request. Seven identical garages are planned to be enabled in the ATLAS TileCal for three independent monitoring systems using three separate sources (one for TLB and two for TLEs).

Because of strict safety requirements the garage is a very complex device (Fig. 13), in which an internal self-sufficient status control is essential.

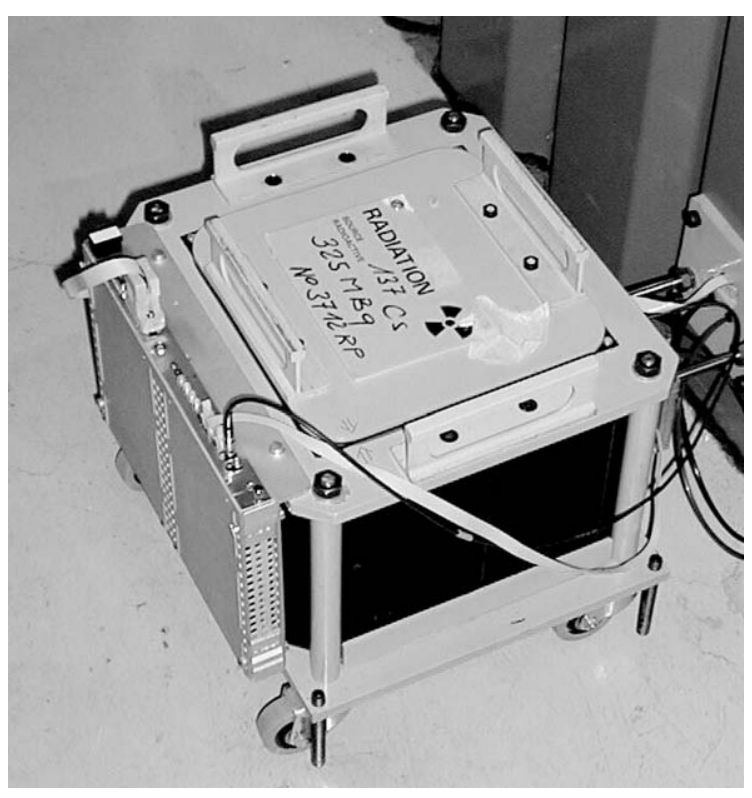

Fig. 13. Control electronics attached to the "garage" at the CERN TileCal test facility. At the top the local information display (LEDs) is visible.

Two types of sensors are implemented in the present garage design: a capsule location sensor and a radiation control monitor.

Early versions of the garage capsule sensor were based on the IRS system. The main advantage of this system is that it can detect, in principle, the 
capsule locked inside the garage at any given time independently of the previous state. On the other hand, the main disadvantage of the IRS approach is its dependence on the liquid purity. In practice, air bubbles gave a false "source presence" recognition too frequently for reliable operation. Furthermore, the necessity of making an open optical channel inside the inner space of the garage poses further problems, so the IRS system turns out to be not suitable.

On the other hand, while the conventional SINbased system satisfies the requirements of reliability, it responds only to changes in frequency, i.e. only the entering or exiting of a capsule is registered, but not the stationary state. To solve this problem, the following improvements were made to the SIN sensor:

- A solenoidal coil was used to increase the interaction of the capsule with the electromagnetic field and, therefore, the sensitivity to frequency shifts was enhanced.

- Special components to stabilize the oscillator's frequency were used.

- A counting method based on frequency variation measurement was implemented.

- The possibility of recalibrating the sensor via CAN_bus was introduced.

The above changes prove to be successful in making the garage SIN circuit more reliable than that based on the IRS technique.

The second type of sensor in the garage is a radiation detector to measure the presence of the real source and to monitor its intensity variation over the time, a function that will be very useful in the future for safety reasons.

A Russian-made type SBM10 Geiger detector with a thin-wall metal case is used (Fig. 14). The detector has dimensions $25 \mathrm{~mm} \times \varnothing 6 \mathrm{~mm}$, operates in the voltage range of $300-400 \mathrm{~V}$, and is capable of withstanding a load rate of up to 700 pulses/s.

To reduce the nominal counting rate, the Geiger detector is embedded in a lead radiation shield, as shown in Fig. 15. With a $9 \mathrm{mCi}{ }^{137} \mathrm{Cs}$ source and a distance between source and detector of about $25 \mathrm{~mm}$, the counting rate is near 500 pulses/s.

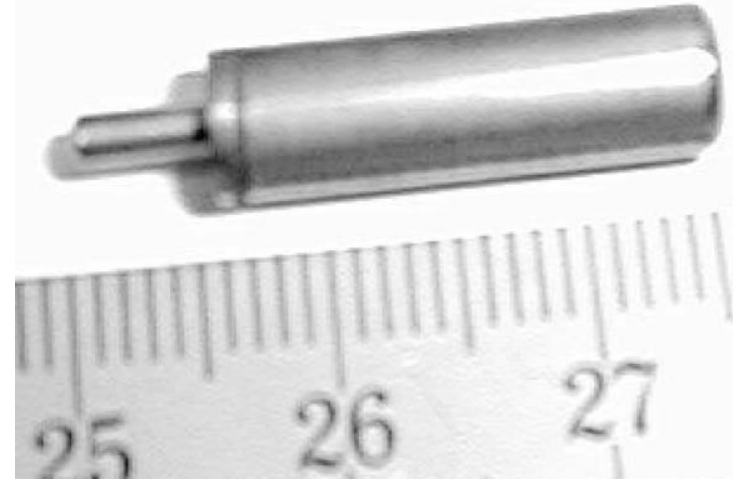

Fig. 14. A Geiger-type SBM10 detector.

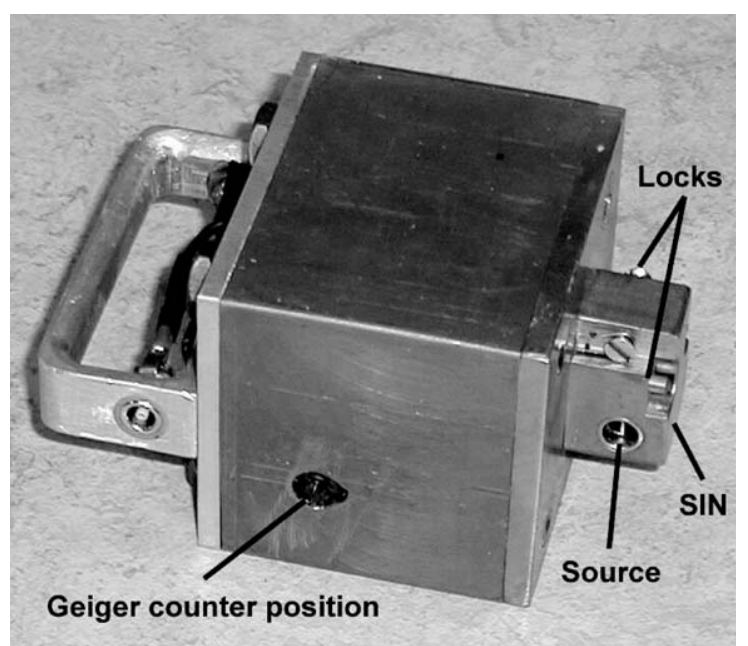

Fig. 15. Location of the Geiger detector, two locks and the SIN sensor on the garage top lid.

A $400 \mathrm{~V}$ DC high-voltage power supply for the Geiger detector is located on the same PCB as the "garage" electronics. It is based on a voltage multiplying principle with a feedback loop, and powered by a $+12 \mathrm{~V}$ supply. A conventional DCDC converter cannot be used because of the intense magnetic field in ATLAS. The Geiger counter is powered only during the measurement period cycle, thus preventing its degradation from the radiation environment.

The two main Geiger counter measurement cycles are:

- A "1 s measurement", used when a capsule's presence is detected in the garage and a 
confirmation of the radioactive source is needed.

- A "128 s measurement", used for monitoring purposes. The data are collected in local memory and are available to be read out. This cycle can be initialized by a sequence of commands over CAN-bus.

The first field tests of the Geiger detector demonstrated a good performance. The capsule detection was found to be $100 \%$ reliable, with no fake signals. As a monitoring device, the counter rate variations were slightly larger than expected. Two main explanations of these variations were found:

- With the current design, the exact position of the source in the garage is determined by the lock's springs, within an accuracy of 4-5 mm. This uncertainty leads to distance variations between the source an the Geiger detector and thus to the variation in measured counts.

- The transport liquid (water) acts as absorber, so its presence or absence around the source capsule can modify the counting rate.

Despite the above uncertainties, the Geiger detector provides better than a $5 \%$ accuracy in monitoring the source activity over a long period, as shown in Fig. 16. Here all available data for the source activity used at the CERN TileCal module instrumentation facility in the year 2001 are given. The data follows the superimposed ${ }^{137} \mathrm{Cs}$ decay curve.

In ATLAS, the monitoring data will be recorded under more stable conditions (with or without water and with the source in the same position) such that the accuracy of the source activity is expected to be even higher. Such precise monitoring provides a check of the integrity of the source and increases the overall safety of the system.

The garage control electronics is organized as a single PCB module with dimensions $240 \times$ $120 \mathrm{~mm}^{2}$ located within $1 \mathrm{~m}$ near the garage and connected to the SIN sensor, to the Geiger detector and to the end switches of the locks. The module sends all information regarding the garage status to a host computer, and is controlled via CAN-bus.

The local display LEDs on the front panel of the module provides the information about the current garage status, including:

- the correct operation of the cabling and power supply;

- the readiness of the CAN-bus communication;

- the status of the SIN sensor: capsule IN/OUT;

- the Geiger counter status: ON, capsule IN/ OUT;

- the status of the Garage locks: Closed/Open.

\section{PS-"pressure sensor"}

Another type of sensor used in the TileCal MonSys is a sensor to control the pressure of the transport medium (water). More than 80 points of pressure measurement within the range of $0-5$ atm are foreseen for the whole system. The main goals of these measurements are:

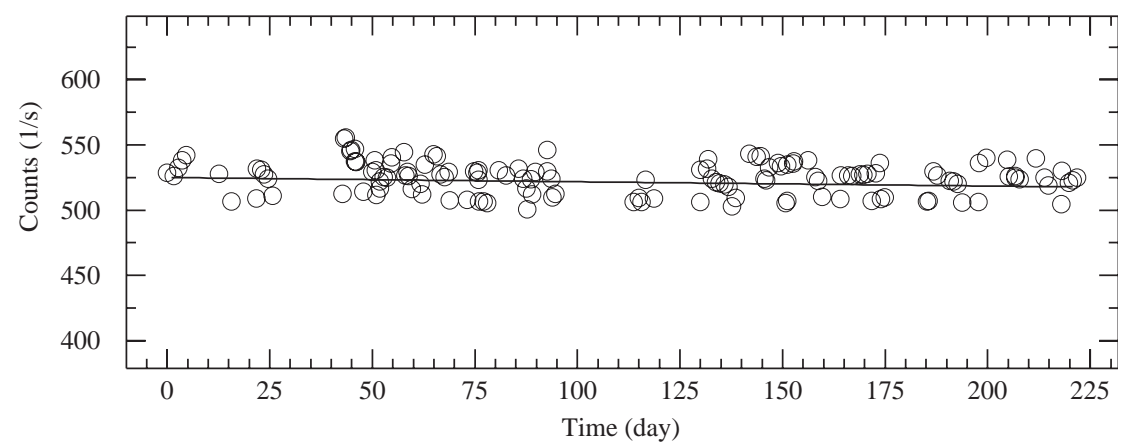

Fig. 16. Garage Geiger detector long-term monitoring data. The line represents the ${ }^{137} \mathrm{Cs}$ decay curve. 
- to check the hermeticity of the system pipeline before it is filled with water, by using a neutral gas under slightly higher than nominal pressure;

- to monitor the process of filling the system with water;

- to check the pressure in different parts of the pipelines according to the program of the calibration run;

- to monitor the process of draining of the system via pressurized gas.

The location of the pressure measurement positions varies; they are placed on tube joints, tee connections, and the ends of tubes. It was decided to use the same pressure-sensing element for all positions, with different body cases depending on the measurement point. An integrated monolithic silicon pressure sensor MPX5700D from Motorola is used as the pressure-sensing element. It has an on-chip piezoresistive transducer with signal conditioning. It is temperature compensated and calibrated with an accuracy better than $2.5 \%$ in the operating range of up to $7 \mathrm{~atm}$.

The operating characteristics and reliability of MPX series pressure sensors are guaranteed if the pressure medium is an inert one, such as dry air, so care was taken to prevent any direct contact between the inner sensor and the water. A polymer (rubber) membrane was used to protect the sensor from water, along with silicon oil as a sealant.

A picture of the pressure sensor is shown in Fig. 17. The sensing element (centre) is fixed between two metal plates (the two pieces on the right) and secured tightly with a rubber O-ring. The inner space of the sensor is filled with silicon oil, covered with a rubber membrane and fixed to the measurement point. On the left of the picture is an example of a measurement point, in this case a calibration tube dead end.

The performance of several sensors was tested in the years 2000 and 2001 and found to be good. In Fig. 18, an example of calibration measurements for the first series of sensors is given. The behaviour of the sensors is found to be linear with increasing pressure over the range $0-5 \mathrm{~atm}$, and the resulting calibration constants are stable over time.

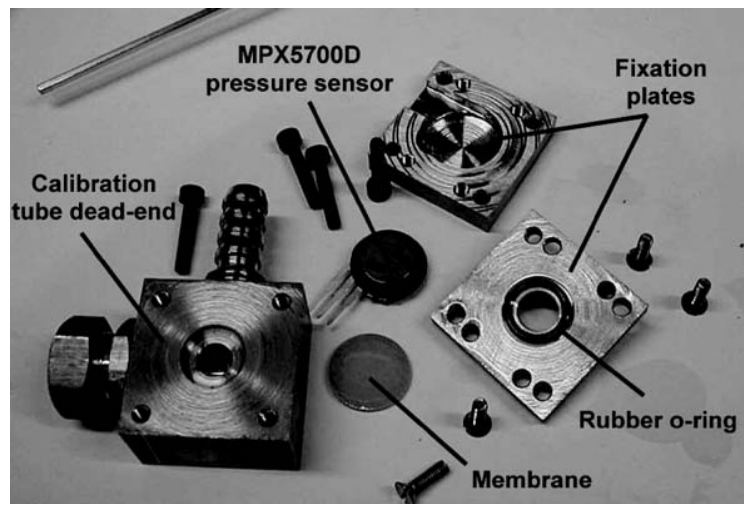

Fig. 17. An example of a pressure sensor case. The sensing base element type MPX5700D is shown in the centre.

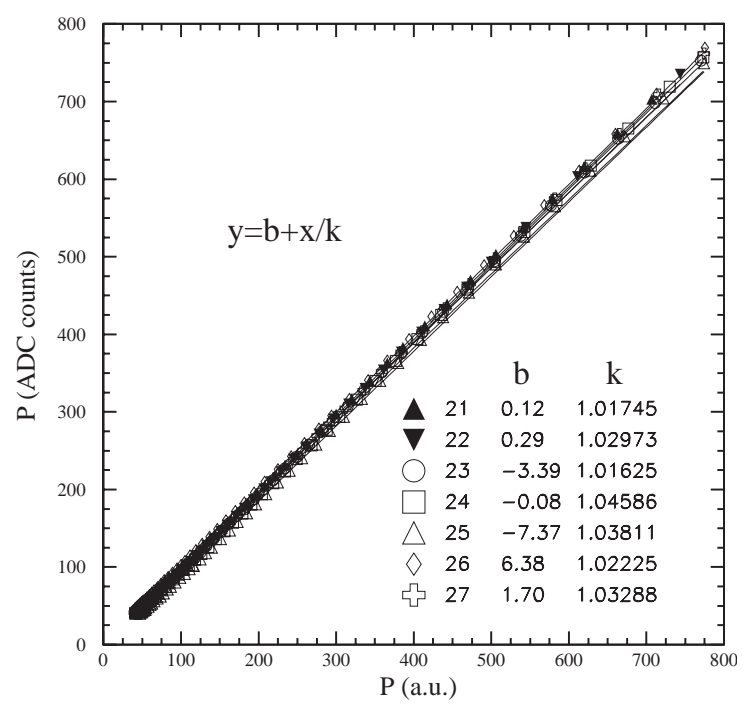

Fig. 18. Calibration data for pressure sensors in the range of 0 $5 \mathrm{~atm}$.

The pressure sensor has a circuit which is compensated for internal temperature variations, providing an accurate, high-level analogue output signal proportional to the applied pressure. The ADC_CAN module, as shown in Fig. 19, utilizes an internal 10-bit successive approximation A/D converter (Philips microcontroller P80CE598) with an on-board CAN-bus controller. The microcontroller has eight inputs multiplexed to one ADC. Analogue signals from the pressure sensors pass through a buffer amplifier before digitization. The output signal of the buffer amplifier is limited to 


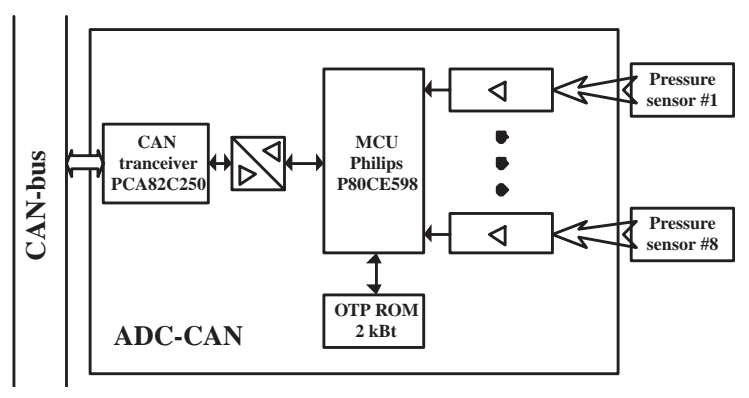

Fig. 19. Schematics of an ADC-CAN module.

$+4 \mathrm{~V}$, while sensor's range varies from 0 to $+5 \mathrm{~V}$. The sensitive range of $0-5 \mathrm{~atm}$ corresponds to sensor output range 0-3.6 V.

\section{Conclusions}

A system of sensors to control the radioactive ${ }^{137} \mathrm{Cs}$ source storage, movement, and working media conditions in the TileCal monitoring system of ATLAS at the LHC has been described. The design, performance, and principles of operation of the sensors have been detailed, covering:

- IRS sensors based on an optical principle, used to detect the source capsule movement in the calibration tubes during initial studies; SIN sensors based on electromagnetic principle, chosen for the use at the final monitoring system;

- a miniature Geiger detector used to check the source capsule integrity and to monitor it during storage;

- a special casing, designed to protect an industrially produced pressure sensor from its surrounding environment;

- the creation and testing of the corresponding electronic modules to acquire the sensor output data;

- online and offline software and algorithms, tested and found to be satisfying the monitoring system requirements;
- information learnt about the operation of the sensors operating under realistic conditions.

The sensor prototypes and the electronic modules have been used over a long period of time at CERN, including the TileCal modules instrumentation facility and the module inter-calibration test bench. All sensors, electronics and modes of operation have been proven to be reliable, and are planned to be used the serial production for the full-scale TileCal MonSys.

\section{Acknowledgements}

The authors are very much grateful to all the Collaboration members who took part in discussions, talks, tests and intensive use of the Cs monitoring system during the past years. All this created the MonSys - an useful tool for the instrumentation and monitoring of the TileCalthe sensor system of which has been described here. Our special gratitude is extended to our technical staff, without their help this work could not have been achieved.

The authors are particularly thankful to R. Teuscher for his help in editing the paper text.

\section{References}

[1] ATLAS Technical Proposal, CERN/LHCC/94-43 LHCC/ P2, December 1994.

[2] Tile Calorimeter Technical Design Report, CERN/LHCC 96-42, December 1996.

[3] G. Blanchot, et al., ATLAS Internal Notes, TILECAL-94044 and TILECAL-94-045, December 1994.

[4] V. Tsupko-Sitnikov, et al., JINR-E13-97-104, April 1997.

[5] A. Karyukhin, et al., ATLAS Internal Note, TILECAL-98134, November 1997.

[6] E. Starchenko, et al., Nucl. Instr. and Meth. A 494 (2002) 381. 